\title{
On a decomposition of integer vectors, II
}

\author{
by \\ ISKANDER Aliev (Warszawa)
}

1. Introduction. Let $\mathbf{n}=\left(n_{1}, \ldots, n_{k+1}\right)$ be an integer vector in $\mathbb{Z}^{k+1}$, $k>1$ and let

$$
h(\mathbf{n})=\max _{i \in\{1, \ldots, k+1\}}\left|n_{i}\right| .
$$

A. Schinzel [7] has proved the following

Theorem A. For every non-zero vector $\mathbf{n} \in \mathbb{Z}^{k+1}$ there exist linearly independent vectors $\mathbf{p}, \mathbf{q} \in \mathbb{Z}^{k+1}$ such that $\mathbf{n}=u \mathbf{p}+v \mathbf{q}$ with $u, v \in \mathbb{Z}$ and

$$
h(\mathbf{p}) h(\mathbf{q}) \leq 2(h(\mathbf{n}))^{1-1 / k} .
$$

The exponent $1-1 / k$ on the right hand side of (1) is the best possible (see [6, Remark 1]). Later S. Chaładus and A. Schinzel [3] have showed that for every non-zero vector $\mathbf{n} \in \mathbb{Z}^{3}$ the inequality (1) can be replaced by

$$
h(\mathbf{p}) h(\mathbf{q})<\frac{2}{\sqrt{3}}(h(\mathbf{n}))^{1 / 2},
$$

where the constant on the right hand side is the best possible. The author [1] has proved a more precise result, which gives estimates that depend on the initial vector $\mathbf{n} \in \mathbb{Z}^{3}$. The aim of this paper is to prove the following

TheOREM 1. For every integer vector $\mathbf{n} \in \mathbb{Z}^{k+1}, k>1$ with $h(\mathbf{n})>1$ there exist linearly independent vectors $\mathbf{p}, \mathbf{q} \in \mathbb{Z}^{k+1}$ such that $\mathbf{n}=u \mathbf{p}+v \mathbf{q}$, $u, v \in \mathbb{Z}$ and

$$
h(\mathbf{p}) h(\mathbf{q})<\frac{2}{(k+1)^{1 / k}}(h(\mathbf{n}))^{1-1 / k} .
$$

For the cases of $k=1$ or $h(\mathbf{n})=1, \mathbf{p}$ and $\mathbf{q}$ can be trivially found with $h(\mathbf{p}) h(\mathbf{q})=1$.

We use the following notation. $\mathrm{By}_{r}^{n}\left(a_{1}, \ldots, a_{n}\right)$ we denote the $n$-dimensional hypercube given by

$$
\mathcal{C}_{r}^{n}\left(a_{1}, \ldots, a_{n}\right)=\left\{\left(x_{1}, \ldots, x_{n}\right) \in \mathbb{R}^{n}:\left|x_{i}-a_{i}\right| \leq r / 2, i \in\{1, \ldots, n\}\right\},
$$

2000 Mathematics Subject Classification: Primary 11H06; Secondary 11D04, 11J25. 
where $a_{i} \in \mathbb{R}, i \in\{1, \ldots, n\}$. We write

$$
H\left(x_{i}=a\right)=\left\{\left(x_{1}, \ldots, x_{n}\right) \in \mathbb{R}^{n}: x_{i}=a\right\}
$$

for $a \in \mathbb{R}, i \in\{1, \ldots, n\}$.

By $V_{n}(K)$ and $\Delta(K)$ we denote the $n$-dimensional volume and the critical determinant of a set $K$ respectively. The convex hull of sets $A_{1}, \ldots, A_{n}$ is denoted by $\operatorname{conv}\left(A_{1}, \ldots, A_{n}\right)$.

2. On the critical determinant of polygons. Let $\mathbf{n}=\left(n_{1}, \ldots, n_{k+1}\right)$ be an integer vector with $k>1, h(\mathbf{n})>1$. We may assume without loss of generality that $0 \leq n_{1} \leq \ldots \leq n_{k+1}$ and $\operatorname{gcd}\left(n_{1}, \ldots, n_{k+1}\right)=1$. Let $n_{i}=n_{i+1}$ for some $i \in\{1, \ldots, n\}$. Consider the vector

$$
\mathbf{n}^{\prime}=\left(n_{1}, \ldots, n_{i}, n_{i+2}, \ldots, n_{k+1}\right) \in \mathbb{Z}^{k} .
$$

If the theorem is true for $\mathbf{n}^{\prime}$ then there exist linearly independent vectors $\mathbf{p}^{\prime}, \mathbf{q}^{\prime} \in \mathbb{Z}^{k}$ such that $\mathbf{n}^{\prime}=u \mathbf{p}^{\prime}+v \mathbf{q}^{\prime}, u, v \in \mathbb{Z}$ and

$$
h\left(\mathbf{p}^{\prime}\right) h\left(\mathbf{q}^{\prime}\right)<\frac{2}{k^{1 /(k-1)}}\left(h\left(\mathbf{n}^{\prime}\right)\right)^{1-1 /(k-1)} .
$$

Suppose $\mathbf{p}^{\prime}=\left(p_{1}^{\prime}, \ldots, p_{k}^{\prime}\right), \mathbf{q}^{\prime}=\left(q_{1}^{\prime}, \ldots, q_{k}^{\prime}\right)$. Put

$$
\mathbf{p}=\left(p_{1}^{\prime}, \ldots, p_{i}^{\prime}, p_{i}^{\prime}, p_{i+1}^{\prime}, \ldots, p_{k}^{\prime}\right), \quad \mathbf{q}=\left(q_{1}^{\prime}, \ldots, q_{i}^{\prime}, q_{i}^{\prime}, q_{i+1}^{\prime}, \ldots, q_{k}^{\prime}\right) .
$$

Then $\mathbf{p}, \mathbf{q}$ are linearly independent vectors in $\mathbb{Z}^{k+1}$ such that $\mathbf{n}=u \mathbf{p}+v \mathbf{q}$ and

$$
h(\mathbf{p}) h(\mathbf{q})<\frac{2}{k^{1 /(k-1)}}(h(\mathbf{n}))^{1-1 /(k-1)} .
$$

Moreover,

$$
\frac{2}{k^{1 /(k-1)}}(h(\mathbf{n}))^{1-1 /(k-1)}<\frac{2}{(k+1)^{1 / k}}(h(\mathbf{n}))^{1-1 / k}
$$

and thus, we may assume without loss of generality that $0<n_{1}<\ldots<$ $n_{k+1}$.

Let $\mathbf{m}=\left(m_{1}, \ldots, m_{k+1}\right)$ be an integer vector such that $\mathbf{m}$ and $\mathbf{n}$ are linearly independent. Consider the polygon

$$
\mathcal{P}: \quad\left|m_{i} y-n_{i} x\right| \leq 1, \quad i \in\{1, \ldots, k+1\}
$$

and sets

$$
\mathcal{H}_{p q r}: \quad\left|m_{i} y-n_{i} x\right| \leq 1, \quad i \in\{p, q, r\},
$$

where $p, q, r \in\{1, \ldots, k+1\}, p<q<r$ and vectors $\left(m_{p}, m_{q}, m_{r}\right),\left(n_{p}, n_{q}, n_{r}\right)$ are linearly independent. Define

$$
\alpha_{i}=\frac{n_{i}}{n_{k+1}}, \quad \delta_{i}=m_{i}-m_{k+1} \alpha_{i}, \quad i \in\{1, \ldots, k\} .
$$

For fixed $p, q, r \in\{1, \ldots, k+1\}$ with $p<q<r$, consider the integer vectors $\left(m_{p}, m_{q}, m_{r}\right)$ and $\left(n_{p}, n_{q}, n_{r}\right)$. Suppose these vectors are linearly indepen- 
dent. Set

$$
\alpha_{p}^{\prime}=\frac{n_{p}}{n_{r}}, \quad \alpha_{q}^{\prime}=\frac{n_{q}}{n_{r}}, \quad \delta_{p}^{\prime}=m_{p}-m_{k+1} \alpha_{p}^{\prime}, \quad \delta_{q}^{\prime}=m_{q}-m_{k+1} \alpha_{q}^{\prime} .
$$

Let $\xi>0$. In [1] we have found a set $\mathcal{B}_{\xi}=\mathcal{B}_{\xi}\left(\alpha_{p}^{\prime}, \alpha_{q}^{\prime}\right) \subset \mathbb{R}^{2}$ such that $\left(\delta_{p}^{\prime}, \delta_{q}^{\prime}\right) \in \mathcal{B}_{\xi}\left(\alpha_{p}^{\prime}, \alpha_{q}^{\prime}\right)$ if and only if

$$
V_{2}\left(\mathcal{H}_{p q r}\right) \geq \frac{4}{n_{r} \xi}
$$

By Lemma 1 of $[1], \mathcal{B}_{\xi}\left(\alpha_{p}^{\prime}, \alpha_{q}^{\prime}\right)$ is a centrally symmetric, convex set. We shall find a set $\mathcal{G}_{p q r} \subset \mathbb{R}^{k}$ such that $\left(\delta_{1}, \ldots, \delta_{k}\right) \in \mathcal{G}_{p q r}$ if and only if

$$
V_{2}\left(\mathcal{H}_{p q r}\right) \geq \frac{4}{n_{k+1} \xi}
$$

In the case $r=k+1$, obviously,

$$
\mathcal{G}_{p q r}=\left\{\left(x_{1}, \ldots, x_{k}\right) \in \mathbb{R}^{k}:\left(x_{p}, x_{q}\right) \in \mathcal{B}_{\xi}\left(\alpha_{p}, \alpha_{q}\right)\right\} .
$$

Let $r<k+1$. Then

$$
\alpha_{p}^{\prime}=\frac{\alpha_{p}}{\alpha_{r}}, \quad \alpha_{q}^{\prime}=\frac{\alpha_{q}}{\alpha_{r}}, \quad \delta_{p}^{\prime}=\delta_{p}-\frac{\alpha_{p}}{\alpha_{r}} \delta_{r}, \quad \delta_{q}^{\prime}=\delta_{q}-\frac{\alpha_{q}}{\alpha_{r}} \delta_{r} .
$$

Moreover, (5) can be rewritten as

$$
V_{2}\left(\mathcal{H}_{p q r}\right) \geq \frac{4}{n_{r} \gamma}, \quad \gamma=\frac{n_{k+1}}{n_{r}} \xi .
$$

Therefore

$$
\mathcal{G}_{p q r}=\left\{\left(x_{1}, \ldots, x_{k}\right) \in \mathbb{R}^{k}:\left(x_{p}-\frac{\alpha_{p}}{\alpha_{r}} x_{r}, x_{q}-\frac{\alpha_{q}}{\alpha_{r}} x_{r}\right) \in \mathcal{B}_{\gamma}\left(\frac{\alpha_{p}}{\alpha_{r}}, \frac{\alpha_{q}}{\alpha_{r}}\right)\right\} .
$$

The above definition, as well as the definition for $r=k+1$ applies only if $\left(m_{p}, m_{q}, m_{r}\right)$ and $\left(n_{p}, n_{q}, n_{r}\right)$ are linearly independent.

Let us find a set $\mathcal{M}_{\xi} \subset \mathbb{R}^{k}$ such that $\left(\delta_{1}, \ldots, \delta_{k}\right) \in \mathcal{M}_{\xi}$ if and only if

$$
\Delta(\mathcal{P}) \geq \frac{1}{n_{k+1} \xi}
$$

By the theorem of K. Mahler (Theorem 5 in [5])

$$
\Delta(\mathcal{P})=\frac{1}{4} \min V_{2}\left(\mathcal{H}_{p q r}\right),
$$

where the minimum on the right is over all $p, q, r \in\{1, \ldots, k+1\}$ such that $p<q<r$ and vectors $\left(m_{p}, m_{q}, m_{r}\right)$ and $\left(n_{p}, n_{q}, n_{r}\right)$ are linearly independent. Therefore

$$
\mathcal{M}_{\xi}=\bigcap \mathcal{G}_{p q r} .
$$

As is easily seen, all sets $\mathcal{G}_{p q r}$ on the right hand side of (6) are centrally symmetric and convex. Thus, $\mathcal{M}_{\xi}$ is centrally symmetric, convex set as well. 
3. Definitions of $E$ and $F$. Define two sequences of sets $\left\{E^{i}\right\}_{i=1}^{k}$ and $\left\{F^{i}\right\}_{i=1}^{k}$ as follows. For $i=1$, let

$$
E^{1}=F^{1}=[-1,1] \subset \mathbb{R}
$$

For $i \in\{2, \ldots, k\}$ set

$$
\begin{aligned}
E_{0}^{i-1} & =\left\{\left(x_{1}, \ldots, x_{i-1}, 0\right) \in \mathbb{R}^{i}:\left(x_{1}, \ldots, x_{i-1}\right) \in E^{i-1}\right\} \\
E_{1}^{i-1} & =\left\{\left(x_{1}, \ldots, x_{i-1}, 1\right) \in \mathbb{R}^{i}:\left(x_{1}, \ldots, x_{i-1}\right) \in \mathcal{C}_{1}^{i-1}(1 / 2, \ldots, 1 / 2)\right\}, \\
F_{0}^{i-1} & =\left\{\left(x_{1}, \ldots, x_{i-1}, 0\right) \in \mathbb{R}^{i}:\left(x_{1}, \ldots, x_{i-1}\right) \in F^{i-1}\right\} \\
F_{1}^{i-1} & =\left\{\left(x_{1}, \ldots, x_{i-1}, 1\right) \in \mathbb{R}^{i}:\left(x_{1}, \ldots, x_{i-1}\right) \in \mathcal{C}_{1}^{i-1}\left(\alpha_{1} / 2, \ldots, \alpha_{i-1} / 2\right)\right\}, \\
E^{i} & =\operatorname{conv}\left(E_{0}^{i-1}, E_{1}^{i-1},-E_{1}^{i-1}\right) \subset \mathbb{R}^{i}, \\
F^{i} & =\operatorname{conv}\left(F_{0}^{i-1}, F_{1}^{i-1},-F_{1}^{i-1}\right) \subset \mathbb{R}^{i} .
\end{aligned}
$$

We write

$$
E_{\lambda}^{i-1}=E^{i} \cap H\left(x_{i}=\lambda\right), \quad F_{\lambda}^{i-1}=F^{i} \cap H\left(x_{i}=\lambda\right), \quad \lambda \in(0,1) .
$$

Define $E=E^{k}$ and $F=F^{k}$. Then $E, F$ are $k$-dimensional polytopes with volumes

$$
V_{k}(E)=2 \int_{0}^{1} V_{k-1}\left(E_{\lambda}^{k-1}\right) d \lambda, \quad V_{k}(F)=2 \int_{0}^{1} V_{k-1}\left(F_{\lambda}^{k-1}\right) d \lambda .
$$

4. The definition and properties of $\widehat{E}$. Consider the set

$$
\widehat{E}=\left\{\left(x_{1}, \ldots, x_{k}\right) \in \mathbb{R}^{k}:\left|x_{i}\right| \leq 1,\left|x_{i}-x_{j}\right| \leq 1, i, j \in\{1, \ldots, k\}, i \neq j\right\} .
$$

It will be proved in Lemma 7 that $\widehat{E}=E$.

LEMma 1.

$$
V_{k}(\widehat{E})=k+1, \quad \Delta(\widehat{E})=(k+1) 2^{-k} .
$$

Proof. H. G. ApSimon [2] (see also [4, Ch. 5, §32]) proved that the body $\mathcal{A} \subset \mathbb{R}^{k}$ defined by the inequalities

$$
\left|x_{i}\right| \leq 1+c_{i}, \quad\left|x_{i}-x_{j}\right| \leq c_{i}+c_{j}-2, \quad i, j \in\{1, \ldots, k\}, i \neq j,
$$

where $c_{i}>1$ for $i \in\{1, \ldots, k\}$, has a unique critical lattice $\Lambda$ with the basis

$$
\left(c_{1}+1,2, \ldots, 2\right),\left(2, c_{2}+1, \ldots, 2\right), \ldots,\left(2,2, \ldots, c_{k}+1\right)
$$

and

$$
d(\Lambda)=\Delta(\mathcal{A})=2^{-k} V_{k}(\mathcal{A})=\left(\prod_{i=1}^{k}\left(c_{i}-1\right)\right)\left(1+2 \sum_{i=1}^{k}\left(c_{i}-1\right)^{-1}\right) .
$$

Put $c_{i}=3$ for $i \in\{1, \ldots, k\}$; then $\mathcal{A}=4 \widehat{E}$ and therefore

$$
V_{k}(\widehat{E})=4^{-k} V_{k}(\mathcal{A})=k+1, \quad \Delta(\widehat{E})=4^{-k} \Delta(\mathcal{A})=(k+1) 2^{-k} .
$$


5. Connection between $E$ and $\widehat{E}$. Let us give another, equivalent definition of $\widehat{E}$. We define a sequence $\left\{\widehat{E}^{i}\right\}_{i=1}^{k}$. For $i=1$ put $\widehat{E}^{1}=[-1,1] \subset$ $\mathbb{R}$. For $i \in\{2, \ldots, k\}$ set

$$
\begin{aligned}
& \widehat{A}^{i}=\left\{\left(x_{1}, \ldots, x_{i}\right) \in \mathbb{R}^{i}:\left(x_{1}, \ldots, x_{i-1}\right) \in \widehat{E}^{i-1}\right\}, \\
& \widehat{B}^{i}=\left\{\left(x_{1}, \ldots, x_{i}\right) \in \mathbb{R}^{i}:\left|x_{i}\right| \leq 1,\left|x_{i}-x_{j}\right| \leq 1, j \in\{1, \ldots, i-1\}\right\}, \\
& \widehat{E}^{i}=\widehat{A}^{i} \cap \widehat{B}^{i} .
\end{aligned}
$$

Then, obviously, $\widehat{E}=\widehat{E}^{k}$.

LEMMA 2. $E \subset \widehat{E}$ and therefore $V_{k}(E) \leq k+1$.

Proof. It suffices to show that $E^{i} \subset \widehat{E}^{i}$ for $i \in\{1, \ldots, k\}$. We use induction on $i$. The case $i=1$ is trivial. Furthermore,

$$
\widehat{E}^{i} \cap H\left(x_{i}=1\right)=E_{1}^{i-1}, \quad \widehat{E}^{i} \cap H\left(x_{i}=-1\right)=-E_{1}^{i-1}
$$

and by the inductive hypothesis $E_{0}^{i-1} \subset \widehat{E}^{i} \cap H\left(x_{i}=0\right)$. Since $\widehat{E}^{i}$ is convex, we obtain $E^{i} \subset \widehat{E}^{i}$.

6. Geometric tools. Let $K_{0}, K_{1}$ be bounded convex sets in $\mathbb{R}^{l-1}, l>1$. Define

$$
\begin{aligned}
& K_{0}^{l-1}=\left\{\left(x_{1}, \ldots, x_{l-1}, 0\right) \in \mathbb{R}^{l}:\left(x_{1}, \ldots, x_{l-1}\right) \in K_{0}\right\}, \\
& K_{1}^{l-1}=\left\{\left(x_{1}, \ldots, x_{l-1}, 1\right) \in \mathbb{R}^{l}:\left(x_{1}, \ldots, x_{l-1}\right) \in K_{1}\right\}, \\
& K_{\lambda}^{l-1}=\operatorname{conv}\left(K_{0}^{l-1}, K_{1}^{l-1}\right) \cap H\left(x_{l}=\lambda\right), \quad \lambda \in[0,1] .
\end{aligned}
$$

Lemma 3. For all $\lambda, \eta \in[0,1]$,

$$
\operatorname{conv}\left(K_{\eta}^{l-1}, K_{1}^{l-1}\right) \cap H\left(x_{l}=\mu\right)=K_{\mu}^{l-1}, \quad \mu=\eta+\lambda(1-\eta) .
$$

Proof. We have

$$
\operatorname{conv}\left(K_{\eta}^{l-1}, K_{1}^{l-1}\right)=\bigcup_{a \in[0,1]}\left\{(1-a) K_{\eta}^{l-1}+a K_{1}^{l-1}\right\} .
$$

From the equation $x_{l}=(1-a) \eta+a=\mu$, we obtain $a=\lambda$ and hence

$$
\operatorname{conv}\left(K_{\eta}^{l-1}, K_{1}^{l-1}\right) \cap H\left(x_{l}=\mu\right)=(1-\lambda) K_{\eta}^{l-1}+\lambda K_{1}^{l-1} .
$$

Furthermore, $K_{\eta}^{l-1}=(1-\eta) K_{0}^{l-1}+\eta K_{1}^{l-1}$ and thus, by the convexity of $K_{1}^{l-1}$, we obtain

$$
\begin{gathered}
\operatorname{conv}\left(K_{\eta}^{l-1}, K_{1}^{l-1}\right) \cap H\left(x_{l}=\mu\right)=(1-\lambda)\left((1-\eta) K_{0}^{l-1}+\eta K_{1}^{l-1}\right)+\lambda K_{1}^{l-1} \\
\quad=(1-\lambda)(1-\eta) K_{0}^{l-1}+(\lambda+\eta(1-\lambda)) K_{1}^{l-1}=(1-\mu) K_{0}^{l-1}+\mu K_{1}^{l-1} .
\end{gathered}
$$


Define

$$
\begin{aligned}
& K_{00}^{l-1}=\left\{\left(x_{1}, \ldots, x_{l-1}, 0,0\right) \in \mathbb{R}^{l+1}:\left(x_{1}, \ldots, x_{l-1}\right) \in K_{0}\right\}, \\
& K_{10}^{l-1}=\left\{\left(x_{1}, \ldots, x_{l-1}, 1,0\right) \in \mathbb{R}^{l+1}:\left(x_{1}, \ldots, x_{l-1}\right) \in K_{1}\right\}, \\
& K_{01}^{l-1}=\left\{\left(x_{1}, \ldots, x_{l-1}, 0,1\right) \in \mathbb{R}^{l+1}:\left(x_{1}, \ldots, x_{l-1}\right) \in K_{1}\right\} .
\end{aligned}
$$

Lemma 4. For every $\lambda \in[0,1]$,

$V_{l-1}\left(\operatorname{conv}\left(K_{00}^{l-1}, K_{10}^{l-1}\right) \cap H\left(x_{l}=\lambda\right)\right)=V_{l-1}\left(\operatorname{conv}\left(K_{00}^{l-1}, K_{01}^{l-1}\right) \cap H\left(x_{l+1}=\lambda\right)\right)$.

Proof. We can obtain $\operatorname{conv}\left(K_{00}^{l-1}, K_{01}^{l-1}\right)$ from $\operatorname{conv}\left(K_{00}^{l-1}, K_{10}^{l-1}\right)$ by exchanging the variables $x_{l}, x_{l+1}$.

For $a \in \mathbb{R}$ define

$$
K_{a 1}^{l-1}=\left\{\left(x_{1}, \ldots, x_{l-1}, a, 1\right) \in \mathbb{R}^{l+1}:\left(x_{1}, \ldots, x_{l-1}\right) \in K_{1}\right\} .
$$

Lemma 5. For all $\lambda, \eta \in[0,1], a \in \mathbb{R}$,

$$
\begin{aligned}
& V_{l-1}\left(\operatorname{conv}\left(\operatorname{conv}\left(K_{00}^{l-1}, K_{10}^{l-1}\right) \cap H\left(x_{l}=\eta\right), K_{a 1}^{l-1}\right) \cap H\left(x_{l+1}=\lambda\right)\right) \\
& \quad=V_{l-1}\left(\operatorname{conv}\left(\operatorname{conv}\left(K_{00}^{l-1}, K_{10}^{l-1}\right) \cap H\left(x_{l}=\eta\right), K_{10}^{l-1}\right) \cap H\left(x_{l}=\mu\right)\right),
\end{aligned}
$$

where $\mu=\eta+\lambda(1-\eta)$.

Proof. Define

$$
\begin{aligned}
L_{\eta} & =\operatorname{conv}\left(K_{00}^{l-1}, K_{10}^{l-1}\right) \cap H\left(x_{l}=\eta\right), \\
M_{\lambda} & =\operatorname{conv}\left(L_{\eta}, K_{a 1}^{l-1}\right) \cap H\left(x_{l+1}=\lambda\right), \\
N_{\mu} & =\operatorname{conv}\left(L_{\eta}, K_{10}^{l-1}\right) \cap H\left(x_{l}=\mu\right) .
\end{aligned}
$$

We show that $V_{l-1}\left(M_{\lambda}\right)=V_{l-1}\left(N_{\mu}\right)$. Consider the translation $T$ :

$$
x_{1}^{\prime}=x_{1}, \ldots, x_{l-1}^{\prime}=x_{l-1}, x_{l}^{\prime}=x_{l}-\eta, x_{l+1}^{\prime}=x_{l+1} .
$$

All volumes are invariant under $T$. Let

$$
L_{\eta} \stackrel{T}{\longrightarrow} U, \quad K_{10}^{l-1} \stackrel{T}{\longrightarrow} V, \quad K_{a 1}^{l-1} \stackrel{T}{\longrightarrow} W .
$$

Therefore

$$
\begin{aligned}
& M_{\lambda} \stackrel{T}{\longrightarrow} Y=\operatorname{conv}(U, W) \cap H\left(x_{l+1}^{\prime}=\lambda\right), \\
& N_{\mu} \stackrel{T}{\longrightarrow} X=\operatorname{conv}(U, V) \cap H\left(x_{l}^{\prime}=\mu-\eta\right) .
\end{aligned}
$$

Consider the sets $U, V, X$, and the affine transformation $\phi$ :

$$
x_{1}^{\prime \prime}=x_{1}^{\prime}, \ldots, x_{l-1}^{\prime \prime}=x_{l-1}^{\prime}, x_{l}^{\prime \prime}=(1-\eta)^{-1} x_{l}^{\prime}, x_{l+1}^{\prime \prime}=x_{l+1}^{\prime} .
$$

Let

$$
U \stackrel{\phi}{\longrightarrow} U_{\phi}, \quad V \stackrel{\phi}{\longrightarrow} V_{\phi}, \quad X \stackrel{\phi}{\longrightarrow} X_{\phi}
$$


Since $U \subset H\left(x_{l}^{\prime}=0\right)$, we have $U=U_{\phi}$. Further, $V_{\phi} \subset H\left(x_{l}^{\prime \prime}=1\right) \cap H\left(x_{l+1}^{\prime \prime}=0\right)$ and

$$
\begin{aligned}
X_{\phi} & =\operatorname{conv}\left(U_{\phi}, V_{\phi}\right) \cap H\left(x_{l}^{\prime \prime}=(1-\eta)^{-1}(\mu-\eta)\right) \\
& =\operatorname{conv}\left(U_{\phi}, V_{\phi}\right) \cap H\left(x_{l}^{\prime \prime}=(1-\eta)^{-1}(\eta+\lambda(1-\eta)-\eta)\right) \\
& =\operatorname{conv}\left(U_{\phi}, V_{\phi}\right) \cap H\left(x_{l}^{\prime \prime}=\lambda\right) .
\end{aligned}
$$

Obviously, $V_{l-1}\left(X_{\phi}\right)=V_{l-1}(X)$. Consider the sets $U, W, Y$, and the affine transformation $\psi$ :

$$
x_{1}^{\prime \prime}=x_{1}^{\prime}, \ldots, x_{l-1}^{\prime \prime}=x_{l-1}^{\prime}, x_{l}^{\prime \prime}=x_{l}^{\prime}+(\eta-a) x_{l+1}^{\prime}, x_{l+1}^{\prime \prime}=x_{l+1}^{\prime} .
$$

Let

$$
U \stackrel{\psi}{\longrightarrow} U_{\psi}, \quad W \stackrel{\psi}{\longrightarrow} W_{\psi}, \quad Y \stackrel{\psi}{\longrightarrow} Y_{\psi} .
$$

Since $U \subset H\left(x_{l}^{\prime}=0\right) \cap H\left(x_{l+1}^{\prime}=0\right)$, we have $U=U_{\psi}$. Further, we have the inclusion $W_{\psi} \subset H\left(x_{l}^{\prime \prime}=0\right) \cap H\left(x_{l+1}^{\prime \prime}=1\right)$ and $Y_{\psi}=\operatorname{conv}\left(U_{\psi}, W_{\psi}\right) \cap$ $H\left(x_{l+1}^{\prime \prime}=\lambda\right)$. Obviously, $V_{l-1}\left(Y_{\psi}\right)=V_{l-1}(Y)$. Lemma 4 with $K_{00}^{l-1}=U=$ $U_{\phi}=U_{\psi}, K_{10}^{l-1}=V_{\phi}, K_{01}^{l-1}=W_{\psi}$ implies $V_{l-1}\left(X_{\phi}\right)=V_{l-1}\left(Y_{\psi}\right)$ and thus $V_{l-1}\left(M_{\lambda}\right)=V_{l-1}\left(N_{\mu}\right)$.

Lemma 6. For all $\lambda, \eta \in[0,1], a \in \mathbb{R}$,

$$
\begin{aligned}
V_{l-1}\left(\operatorname { c o n v } \left(\operatorname{conv}\left(K_{00}^{l-1}, K_{10}^{l-1}\right)\right.\right. & \left.\left.\cap H\left(x_{l}=\eta\right), K_{a 1}^{l-1}\right) \cap H\left(x_{l+1}=\lambda\right)\right) \\
& =V_{l-1}\left(\operatorname{conv}\left(K_{0}^{l-1}, K_{1}^{l-1}\right) \cap H\left(x_{l}=\mu\right)\right),
\end{aligned}
$$

where $\mu=\eta+\lambda(1-\eta)$.

Proof. By Lemma 5 it is enough to prove that

$$
\begin{aligned}
V_{l-1}\left(\operatorname { c o n v } \left(\operatorname{conv}\left(K_{00}^{l-1}, K_{10}^{l-1}\right)\right.\right. & \left.\left.\cap H\left(x_{l}=\eta\right), K_{10}^{l-1}\right) \cap H\left(x_{l}=\mu\right)\right) \\
& =V_{l-1}\left(\operatorname{conv}\left(K_{0}^{l-1}, K_{1}^{l-1}\right) \cap H\left(x_{l}=\mu\right)\right) .
\end{aligned}
$$

Since $K_{00}^{l-1}, K_{10}^{l-1} \subset H\left(x_{l+1}=0\right)$, we have

$$
\begin{aligned}
V_{l-1}\left(\operatorname { c o n v } \left(\operatorname{conv}\left(K_{00}^{l-1}, K_{10}^{l-1}\right)\right.\right. & \left.\left.\cap H\left(x_{l}=\eta\right), K_{10}^{l-1}\right) \cap H\left(x_{l}=\mu\right)\right) \\
= & V_{l-1}\left(\operatorname{conv}\left(K_{\eta}^{l-1}, K_{1}^{l-1}\right) \cap H\left(x_{l}=\mu\right)\right) \\
& =V_{l-1}\left(\operatorname{conv}\left(K_{0}^{l-1}, K_{1}^{l-1}\right) \cap H\left(x_{l}=\mu\right)\right),
\end{aligned}
$$

where the last equality is valid in view of Lemma 3.

\section{A property of $E$}

Lemma 7. For every $\lambda \in[0,1]$,

(i) $E_{\lambda}^{n}=\operatorname{conv}\left(E_{0}^{n}, E_{1}^{n}\right) \cap H\left(x_{n+1}=\lambda\right)$,

(ii) $V_{n}\left(E_{\lambda}^{n}\right)=-n \lambda+n+1, n \in\{1, \ldots, k-1\}$.

Moreover, $V_{k}(E)=k+1$ and thus $E=\widehat{E}$. 
Proof. We use induction on $n$. The case $n=1$ is trivial. The affine transformation

$$
x_{1}^{\prime}=x_{1}, \ldots, x_{n-1}^{\prime}=x_{n-1}, x_{n}^{\prime}=x_{n}-\frac{1}{2} x_{n+1}, x_{n+1}^{\prime}=x_{n+1}
$$

does not change the volume of a body and the volume of its intersection with any hyperplane $H\left(x_{n+1}=a\right)$. Thus, we may assume without loss of generality that

$$
E_{1}^{n}=\left\{\left(x_{1}, \ldots, x_{n}, 1\right) \in \mathbb{R}^{n+1}:\left(x_{1}, \ldots, x_{n}\right) \in \mathcal{C}_{1}^{n}(1 / 2, \ldots, 1 / 2,0)\right\} .
$$

Therefore

$$
\begin{aligned}
& V_{n}\left(\operatorname{conv}\left(E_{0}^{n}, E_{1}^{n}\right) \cap H\left(x_{n+1}=\lambda\right)\right) \\
& \quad=2 \int_{0}^{1-\lambda / 2} V_{n-1}\left(\operatorname{conv}\left(E_{0}^{n}, E_{1}^{n}\right) \cap H\left(x_{n+1}=\lambda\right) \cap H\left(x_{n}=\xi\right)\right) d \xi .
\end{aligned}
$$

Let us show that $\operatorname{conv}\left(E_{0}^{n}, E_{1}^{n}\right) \cap H\left(x_{n+1}=\lambda\right) \cap H\left(x_{n}=\xi\right)$, where $\lambda \in(0,1)$, contains the set

$$
E_{1}(\xi)=\operatorname{conv}\left(E_{0}^{n} \cap H\left(x_{n}=0\right), E_{1}^{n} \cap H\left(x_{n}=\xi / \lambda\right)\right) \cap H\left(x_{n+1}=\lambda\right)
$$

for $0 \leq \xi \leq \lambda / 2$, and

$$
E_{2}(\xi)=\operatorname{conv}\left(E_{0}^{n} \cap H\left(x_{n}=\eta\right), E_{1}^{n} \cap H\left(x_{n}=1 / 2\right)\right) \cap H\left(x_{n+1}=\lambda\right)
$$

for $\lambda / 2<\xi \leq 1-\lambda / 2, \eta=(\xi-\lambda / 2) /(1-\lambda)$. Let $0 \leq \xi \leq \lambda / 2$ and $\mathbf{x}=\left(x_{1}, \ldots, x_{n+1}\right) \in E_{1}(\xi)$. Then there exist $\mathbf{x}^{\prime}=\left(x_{1}^{\prime}, \ldots, x_{n-1}^{\prime}, 0,0\right) \in$ $E_{0}^{n} \cap H\left(x_{n}=0\right)$ and $\mathbf{x}^{\prime \prime}=\left(x_{1}^{\prime \prime}, \ldots, x_{n-1}^{\prime \prime}, \xi / \lambda, 1\right) \in E_{1}^{n} \cap H\left(x_{n}=\xi / \lambda\right)$ such that

$$
\mathbf{x}=(1-a) \mathbf{x}^{\prime}+a \mathbf{x}^{\prime \prime}, \quad a \in(0,1) .
$$

Since $x_{n+1}=(1-a) x_{n+1}^{\prime}+a x_{n+1}^{\prime \prime}=\lambda$, we obtain $a=\lambda$. Furthermore,

$$
x_{n}=(1-\lambda) x_{n}^{\prime}+\lambda x_{n}^{\prime \prime}=\xi .
$$

Since $E_{0}^{n} \cap H\left(x_{n}=0\right) \subset E_{0}^{n}$ and $E_{1}^{n} \cap H\left(x_{n}=\xi / \lambda\right) \subset E_{1}^{n}$, we have $\mathbf{x}^{\prime} \in E_{0}^{n}$, $\mathbf{x}^{\prime \prime} \in E_{1}^{n}$ and thus

$$
\mathbf{x} \in \operatorname{conv}\left(E_{0}^{n}, E_{1}^{n}\right) \cap H\left(x_{n+1}=\lambda\right) \cap H\left(x_{n}=\xi\right) .
$$

By similar arguments, the case $\lambda / 2<\xi \leq 1-\lambda / 2$ can be settled as well. Therefore

$$
V_{n}\left(\operatorname{conv}\left(E_{0}^{n}, E_{1}^{n}\right) \cap H\left(x_{n+1}=\lambda\right)\right) \geq 2 I_{1}+2 I_{2},
$$

where

$$
I_{1}=\int_{0}^{\lambda / 2} V_{n-1}\left(E_{1}(\xi)\right) d \xi, \quad I_{2}=\int_{\lambda / 2}^{1-\lambda / 2} V_{n-1}\left(E_{2}(\xi)\right) d \xi
$$


Put $l=n, K_{0}=E^{n-1}, K_{1}=\mathcal{C}_{1}^{n-1}(1 / 2, \ldots, 1 / 2)$. Then in view of part (i) of the inductive hypothesis we have, in the notation of Section 6 ,

and

$$
K_{0}^{n-1}=E_{0}^{n-1}, \quad K_{1}^{n-1}=E_{1}^{n-1}, \quad K_{\lambda}^{n-1}=E_{\lambda}^{n-1}, \quad \lambda \in[0,1]
$$

$$
K_{00}^{n-1}=E_{0}^{n} \cap H\left(x_{n}=0\right), \quad K_{10}^{n-1}=E_{0}^{n} \cap H\left(x_{n}=1\right) .
$$

Assume $\lambda \in(0,1)$, and consider the integral $I_{1}$. Put $\eta=0, a=\xi / \lambda$. Then

$$
\operatorname{conv}\left(K_{00}^{n-1}, K_{10}^{n-1}\right) \cap H\left(x_{n}=\eta\right)=K_{00}^{n-1}, \quad K_{a 1}^{n-1}=E_{1}^{n} \cap H\left(x_{n}=\xi / \lambda\right)
$$

and by Lemma 6 we obtain

$$
\begin{aligned}
V_{n-1}\left(E_{1}(\xi)\right) & =V_{n-1}\left(\operatorname{conv}\left(E_{0}^{n-1}, E_{1}^{n-1}\right) \cap H\left(x_{n}=\lambda\right)\right)=V_{n-1}\left(E_{\lambda}^{n-1}\right) \\
& =(1-n) \lambda+n,
\end{aligned}
$$

where the last two equalities are valid by the inductive hypothesis. Therefore

$$
I_{1}=((1-n) \lambda+n) \int_{0}^{\lambda / 2} d \xi=\frac{\lambda}{2}((1-n) \lambda+n) .
$$

Consider the integral $I_{2}$. Put $\eta=(\xi-\lambda / 2) /(1-\lambda), a=1 / 2$. Then

$$
\begin{gathered}
\operatorname{conv}\left(K_{00}^{n-1}, K_{10}^{n-1}\right) \cap H\left(x_{n}=\eta\right)=E_{0}^{n} \cap H\left(x_{n}=\eta\right), \\
K_{a 1}^{n-1}=E_{1}^{n} \cap H\left(x_{n}=1 / 2\right)
\end{gathered}
$$

and by Lemma 6 we obtain

$$
V_{n-1}\left(E_{2}(\xi)\right)=V_{n-1}\left(\operatorname{conv}\left(E_{0}^{n-1}, E_{1}^{n-1}\right) \cap H\left(x_{n}=\mu\right)\right)=V_{n-1}\left(E_{\mu}^{n-1}\right),
$$

where $\mu=\eta+\lambda(1-\eta)=\xi+\lambda / 2$. By the inductive hypothesis

Therefore

$$
V_{n-1}\left(E_{\mu}^{n-1}\right)=(1-n) \mu+n=(1-n)(\xi+\lambda / 2)+n .
$$

$$
\begin{aligned}
I_{2} & =\int_{\lambda / 2}^{1-\lambda / 2}((1-n)(\xi+\lambda / 2)+n) d \xi \\
& =(1-n) \int_{\lambda / 2}^{1-\lambda / 2} \xi d \xi+((1-n) \lambda / 2+n) \int_{\lambda / 2}^{1-\lambda / 2} d \xi \\
& =(1-n)(1-\lambda) / 2+(1-n)(1-\lambda) \lambda / 2+n(1-\lambda) .
\end{aligned}
$$

Thus

$$
\begin{aligned}
2 I_{1}+ & 2 I_{2} \\
& =\lambda((1-n) \lambda+n)+(1-n)(1-\lambda)+\lambda(1-n)(1-\lambda)+2 n(1-\lambda) \\
& =\lambda^{2}-n \lambda^{2}+n \lambda+1-\lambda-n+n \lambda+\lambda-\lambda^{2}-n \lambda+n \lambda^{2}+2 n-2 n \lambda \\
& =-n \lambda+n+1 .
\end{aligned}
$$


Therefore

$$
V_{n}\left(\operatorname{conv}\left(E_{0}^{n}, E_{1}^{n}\right) \cap H\left(x_{n+1}=\lambda\right)\right) \geq-n \lambda+n+1, \quad \lambda \in(0,1) .
$$

Consider the case $\lambda=0$. By the inductive hypothesis

$$
\begin{aligned}
V_{n}\left(\operatorname{conv}\left(E_{0}^{n}, E_{1}^{n}\right) \cap H\left(x_{n+1}=0\right)\right) & =V_{n}\left(E_{0}^{n}\right)=2 \int_{0}^{1} V_{n-1}\left(E_{\lambda}^{n-1}\right) d \lambda \\
& =2 \int_{0}^{1}((1-n) \lambda+n) d \lambda=n+1 .
\end{aligned}
$$

For the case of $\lambda=1$ we have

$$
V_{n}\left(\operatorname{conv}\left(E_{0}^{n}, E_{1}^{n}\right) \cap H\left(x_{n+1}=1\right)\right)=V_{n}\left(E_{1}^{n}\right)=1 .
$$

Moreover, in view of the inclusion

$$
\operatorname{conv}\left(E_{0}^{n}, E_{1}^{n}\right) \cap H\left(x_{n+1}=\lambda\right) \subset E_{\lambda}^{n}, \quad \lambda \in[0,1],
$$

we have

$$
V_{n}\left(E_{\lambda}^{n}\right) \geq-n \lambda+n+1, \quad \lambda \in(0,1)
$$

and obviously (ii) implies (i). Suppose there exists $\lambda \in(0,1)$, such that $V_{n}\left(E_{\lambda}^{n}\right)>-n \lambda+n+1$. Then, by the continuity of the function $f(\lambda)=$ $V_{n}\left(E_{\lambda}^{n}\right)$ on $(0,1)$, we obtain

$$
V_{n+1}\left(E^{n+1}\right)=2 \int_{0}^{1} V_{n}\left(E_{\lambda}^{n}\right) d \lambda>2 \int_{0}^{1}(-n \lambda+n+1) d \lambda=n+2 .
$$

But Lemma 2 gives $V_{n+1}\left(E^{n+1}\right) \leq n+2$, a contradiction. Therefore, $V_{n}\left(E_{\lambda}^{n}\right)$ $=-n \lambda+n+1$ for every $\lambda \in[0,1]$. Finally,

$$
V_{k}(E)=2 \int_{0}^{1} V_{k-1}\left(E_{\lambda}^{k-1}\right) d \lambda=k+1
$$

and therefore $E=\widehat{E}$.

Remark. The equality $E=\widehat{E}$ has been proved by A. Schinzel, by a different argument.

\section{A property of $F$}

Lemma 8. For every $\lambda \in[0,1]$,

$$
V_{n}\left(\operatorname{conv}\left(F_{0}^{n}, F_{1}^{n}\right) \cap H\left(x_{n+1}=\lambda\right)\right) \geq V_{n}\left(E_{\lambda}^{n}\right), \quad n \in\{1, \ldots, k-1\} .
$$

Moreover, $V_{k}(F) \geq V_{k}(E)=k+1$.

Proof. We use induction on $n$. The case $n=1$ is trivial. The affine transformation

$$
x_{1}^{\prime}=x_{1}, \ldots, x_{n-1}^{\prime}=x_{n-1}, x_{n}^{\prime}=x_{n}-\frac{\alpha_{n}}{2} x_{n+1}, x_{n+1}^{\prime}=x_{n+1}
$$


does not change the volume of a body, and the volume of its intersection with any hyperplane $H\left(x_{n+1}=a\right)$. Thus, we may assume without loss of generality that

$$
F_{1}^{n}=\left\{\left(x_{1}, \ldots, x_{n}, 1\right) \in \mathbb{R}^{n+1}:\left(x_{1}, \ldots, x_{n}\right) \in \mathcal{C}_{1}^{n}\left(\alpha_{1} / 2, \ldots, \alpha_{n-1} / 2,0\right)\right\} .
$$

Therefore

$$
\begin{aligned}
& V_{n}\left(\operatorname{conv}\left(F_{0}^{n}, F_{1}^{n}\right) \cap H\left(x_{n+1}=\lambda\right)\right) \\
& \quad=2 \int_{0}^{1-\lambda / 2} V_{n-1}\left(\operatorname{conv}\left(F_{0}^{n}, F_{1}^{n}\right) \cap H\left(x_{n+1}=\lambda\right) \cap H\left(x_{n}=\xi\right)\right) d \xi .
\end{aligned}
$$

Define

$$
F_{00}^{n-1}=\left\{\left(x_{1}, \ldots, x_{n-1}, 0,0\right) \in \mathbb{R}^{n+1}:\left(x_{1}, \ldots, x_{n-1}\right) \in F^{n-1}\right\} .
$$

Then, in particular,

$$
F_{00}^{n-1}=\left\{\left(x_{1}, \ldots, x_{n}, 0\right) \in \mathbb{R}^{n+1}:\left(x_{1}, \ldots, x_{n}\right) \in F_{0}^{n-1}\right\} .
$$

Arguments similar to ones of Section 7 show that $\operatorname{conv}\left(F_{0}^{n}, F_{1}^{n}\right) \cap$ $H\left(x_{n+1}=\lambda\right) \cap H\left(x_{n}=\xi\right)$, where $\lambda \in(0,1)$, contains the set

$$
F_{1}(\xi)=\operatorname{conv}\left(F_{00}^{n-1}, F_{1}^{n} \cap H\left(x_{n}=\xi / \lambda\right)\right) \cap H\left(x_{n+1}=\lambda\right)
$$

for $0 \leq \xi \leq \lambda / 2$, and

$$
\begin{array}{r}
F_{2}(\xi)=\operatorname{conv}\left(\operatorname{conv}\left(F_{00}^{n-1}, F_{0}^{n} \cap H\left(x_{n}=1\right)\right) \cap H\left(x_{n}=\eta\right), F_{1}^{n} \cap H\left(x_{n}=1 / 2\right)\right) \\
\cap H\left(x_{n+1}=\lambda\right)
\end{array}
$$

for $\lambda / 2<\xi \leq 1-\lambda / 2, \eta=(\xi-\lambda / 2) /(1-\lambda)$. Therefore

$$
V_{n}\left(\operatorname{conv}\left(F_{0}^{n}, F_{1}^{n}\right) \cap H\left(x_{n+1}=\lambda\right)\right) \geq 2 J_{1}+2 J_{2},
$$

where

$$
J_{1}=\int_{0}^{\lambda / 2} V_{n-1}\left(F_{1}(\xi)\right) d \xi, \quad J_{2}=\int_{\lambda / 2}^{1-\lambda / 2} V_{n-1}\left(F_{2}(\xi)\right) d \xi .
$$

Put $l=n, K_{0}=F^{n-1}, K_{1}=\mathcal{C}_{1}^{n-1}\left(\alpha_{1} / 2, \ldots, \alpha_{n-1} / 2\right)$. Then, in the notation of Section 6, we have

$$
\begin{aligned}
& K_{0}^{n-1}=F_{0}^{n-1}, \quad K_{1}^{n-1}=F_{1}^{n-1}, \\
& K_{\lambda}^{n-1}=\operatorname{conv}\left(F_{0}^{n-1}, F_{1}^{n-1}\right) \cap H\left(x_{n}=\lambda\right), \quad \lambda \in[0,1], \\
& K_{00}^{n-1}=F_{00}^{n-1}, \quad K_{10}^{n-1}=F_{0}^{n} \cap H\left(x_{n}=1\right) .
\end{aligned}
$$
Then

Assume $\lambda \in(0,1)$, and consider the integral $J_{1}$. Put $\eta=0, a=\xi / \lambda$.

$$
\operatorname{conv}\left(K_{00}^{n-1}, K_{10}^{n-1}\right) \cap H\left(x_{n}=\eta\right)=F_{00}^{n-1}, \quad K_{a 1}^{n-1}=F_{1}^{n} \cap H\left(x_{n}=\xi / \lambda\right)
$$

and by Lemma 6 we obtain

$$
V_{n-1}\left(F_{1}(\xi)\right)=V_{n-1}\left(\operatorname{conv}\left(F_{0}^{n-1}, F_{1}^{n-1}\right) \cap H\left(x_{n}=\lambda\right)\right) \geq V_{n-1}\left(E_{\lambda}^{n-1}\right),
$$


where the last inequality is valid by the inductive hypothesis. Therefore

$$
J_{1} \geq V_{n-1}\left(E_{\lambda}^{n-1}\right) \int_{0}^{\lambda / 2} d \xi .
$$

Consider the integral $J_{2}$. Put $\eta=(\xi-\lambda / 2) /(1-\lambda), a=1 / 2$. Then $\operatorname{conv}\left(K_{00}^{n-1}, K_{10}^{n-1}\right) \cap H\left(x_{n}=\eta\right)=\operatorname{conv}\left(F_{00}^{n-1}, F_{0}^{n} \cap H\left(x_{n}=1\right)\right) \cap H\left(x_{n}=\eta\right)$,

$$
K_{a 1}^{n-1}=F_{1}^{n} \cap H\left(x_{n}=1 / 2\right)
$$

and by Lemma 6 we obtain

$$
V_{n-1}\left(F_{2}(\xi)\right)=V_{n-1}\left(\operatorname{conv}\left(F_{0}^{n-1}, F_{1}^{n-1}\right) \cap H\left(x_{n}=\mu\right)\right) \geq V_{n-1}\left(E_{\mu}^{n-1}\right),
$$

where $\mu=\xi+\lambda / 2$ and the last inequality is valid by the inductive hypothesis. Therefore

$$
J_{2} \geq \int_{\lambda / 2}^{1-\lambda / 2} V_{n-1}\left(E_{\xi+\lambda / 2}^{n-1}\right) d \xi
$$

and

$$
\begin{aligned}
& V_{n}\left(\operatorname{conv}\left(F_{0}^{n}, F_{1}^{n}\right) \cap H\left(x_{n+1}=\lambda\right)\right) \\
& \quad \geq 2 J_{1}+2 J_{2} \geq 2 V_{n-1}\left(E_{\lambda}^{n-1}\right) \int_{0}^{\lambda / 2} d \xi+2 \int_{\lambda / 2}^{1-\lambda / 2} V_{n-1}\left(E_{\xi+\lambda / 2}^{n-1}\right) d \xi \\
& \quad=-n \lambda+n+1=V_{n}\left(E_{\lambda}^{n}\right), \quad \lambda \in(0,1) .
\end{aligned}
$$

Consider the case $\lambda=0$. Note that

$$
\operatorname{conv}\left(F_{0}^{n-1}, F_{1}^{n-1}\right) \cap H\left(x_{n}=\lambda\right) \subset F_{\lambda}^{n-1}, \quad \lambda \in[0,1],
$$

thus, by the inductive hypothesis,

$$
\begin{aligned}
& V_{n}\left(\operatorname{conv}\left(F_{0}^{n}, F_{1}^{n}\right) \cap H\left(x_{n+1}=0\right)\right)=V_{n}\left(F_{0}^{n}\right)=2 \int_{0}^{1} V_{n-1}\left(F_{\lambda}^{n-1}\right) d \lambda \\
& \quad \geq 2 \int_{0}^{1} V_{n-1}\left(\operatorname{conv}\left(F_{0}^{n-1}, F_{1}^{n-1}\right) \cap H\left(x_{n}=\lambda\right)\right) d \lambda \geq 2 \int_{0}^{1} V_{n-1}\left(E_{\lambda}^{n-1}\right) d \lambda \\
& \quad=2 \int_{0}^{1}((1-n) \lambda+n) d \lambda=n+1 .
\end{aligned}
$$

In the case $\lambda=1$ we have

$$
V_{n}\left(\operatorname{conv}\left(F_{0}^{n}, F_{1}^{n}\right) \cap H\left(x_{n+1}=1\right)\right)=V_{n}\left(F_{1}^{n}\right)=1 .
$$

Furthermore,

$$
\operatorname{conv}\left(F_{0}^{n}, F_{1}^{n}\right) \cap H\left(x_{n+1}=\lambda\right) \subset F_{\lambda}^{n}, \quad \lambda \in[0,1],
$$


thus, finally,

$$
\begin{aligned}
V_{k}(F) & =2 \int_{0}^{1} V_{k-1}\left(F_{\lambda}^{k-1}\right) d \lambda \\
& \geq 2 \int_{0}^{1} V_{k-1}\left(\operatorname{conv}\left(F_{0}^{k-1}, F_{1}^{k-1}\right) \cap H\left(x_{k}=\lambda\right)\right) d \lambda \\
& \geq 2 \int_{0}^{1} V_{k-1}\left(E_{\lambda}^{k-1}\right) d \lambda=V_{k}(E)=k+1
\end{aligned}
$$

\section{Properties of $\mathcal{G}_{p q r}$}

LEMma 9. For all $p, q \in\{1, \ldots, k\}, p<q$ and $r=k+1$ the set $\mathcal{G}_{p q r}$, if defined (see p. 375), contains points $\left(x_{1}, \ldots, x_{k}\right) \in \mathbb{R}^{k}$ for which one of the following conditions is valid:

(i) $\left(x_{p}, x_{q}\right)= \pm(\xi, 0)$,

(ii) $\left(x_{p}, x_{q}\right)= \pm\left(\left(\alpha_{p} / 2 \pm 1 / 2\right) \xi, 0\right)$,

(iii) $\left(x_{p}, x_{q}\right)= \pm\left(\left(\alpha_{p} / 2 \pm 1 / 2\right) \xi, \xi\right)$,

(iv) $\left(x_{p}, x_{q}\right)= \pm\left(\left(\alpha_{p} / 2 \pm 1 / 2\right) \xi,\left(\alpha_{q} / 2 \pm 1 / 2\right) \xi\right)$,

for any combination of \pm .

Proof. By the definition of $\mathcal{G}_{p q r}$ we have to check the inclusion

$$
\left(x_{p}, x_{q}\right) \in \mathcal{B}_{\xi}\left(\alpha_{p}, \alpha_{q}\right)
$$

for all cases (i)-(iv). It is easy to verify by using formulae (8)-(13) of [1] that $\mathcal{B}_{\xi}\left(\alpha_{p}, \alpha_{q}\right)$ contains the centrally symmetric, convex octagon $\mathcal{O}_{\xi}\left(\alpha_{p}, \alpha_{q}\right)$ with vertices

$$
\begin{array}{ll} 
\pm P^{1}= \pm\left(\xi, \xi\left(1-\alpha_{q}\right) /\left(1-\alpha_{p}\right)\right), & \pm P^{2}= \pm\left(\xi\left(1+\alpha_{p}\right) /\left(1+\alpha_{q}\right), \xi\right), \\
\pm P^{3}= \pm\left(-\xi\left(1-\alpha_{p}\right) /\left(1+\alpha_{q}\right), \xi\right), & \pm P^{4}= \pm\left(-\xi, \xi\left(1-\alpha_{q}\right) /\left(1+\alpha_{p}\right)\right) .
\end{array}
$$

In cases (i)-(iii) the inclusion $\left(x_{p}, x_{q}\right) \in \mathcal{O}_{\xi}\left(\alpha_{p}, \alpha_{q}\right)$ is obviously valid and therefore (7) is true. Consider case (iv). Since case (iii) is proved and $\left(\alpha_{q} / 2+\right.$ $1 / 2) \xi \leq \xi$, we have

$$
\pm\left(\left(\alpha_{p} / 2 \pm 1 / 2\right) \xi,\left(\alpha_{q} / 2+1 / 2\right) \xi\right) \in \mathcal{O}_{\xi}\left(\alpha_{p}, \alpha_{q}\right) .
$$

Since $\left|\left(\alpha_{q} / 2-1 / 2\right) \xi\right| \leq \min \left(\left|P_{y}^{1}\right|,\left|P_{y}^{4}\right|\right)$ the points $\pm\left(\left(\alpha_{p} / 2 \pm 1 / 2\right) \xi,\left(\alpha_{q} / 2-\right.\right.$ $1 / 2) \xi$ ) lie in the rectangle with vertices $P^{1}, P^{4},-P^{1},-P^{4}$ and therefore

$$
\pm\left(\left(\alpha_{p} / 2+1 / 2\right) \xi,\left(\alpha_{q} / 2-1 / 2\right) \xi\right) \in \mathcal{O}_{\xi}\left(\alpha_{p}, \alpha_{q}\right) \text {. }
$$

LEMma 10. For all $p, q, r \in\{1, \ldots, k\}, p<q<r$, the set $\mathcal{G}_{p q r}$, if defined, contains points $\left(x_{1}, \ldots, x_{k}\right) \in \mathbb{R}^{k}$ for which one of the following conditions is valid: 
(i) $\left(x_{p}, x_{q}, x_{r}\right)= \pm(\xi, 0,0)$,

(ii) $\left(x_{p}, x_{q}, x_{r}\right)= \pm\left(\left(\alpha_{p} / 2 \pm 1 / 2\right) \xi, 0,0\right)$,

(iii) $\left(x_{p}, x_{q}, x_{r}\right)= \pm\left(\left(\alpha_{p} / 2 \pm 1 / 2\right) \xi, \xi, 0\right)$,

(iv) $\left(x_{p}, x_{q}, x_{r}\right)= \pm\left(\left(\alpha_{p} / 2 \pm 1 / 2\right) \xi,\left(\alpha_{q} / 2 \pm 1 / 2\right) \xi, 0\right)$,

(v) $\left(x_{p}, x_{q}, x_{r}\right)= \pm\left(\left(\alpha_{p} / 2 \pm 1 / 2\right) \xi,\left(\alpha_{q} / 2 \pm 1 / 2\right) \xi, \xi\right)$,

(vi) $\left(x_{p}, x_{q}, x_{r}\right)= \pm\left(\left(\alpha_{p} / 2 \pm 1 / 2\right) \xi,\left(\alpha_{q} / 2 \pm 1 / 2\right) \xi,\left(\alpha_{r} / 2 \pm 1 / 2\right) \xi\right)$,

for any combination of \pm .

Proof. By the definition of $\mathcal{G}_{p q r}$ we have to check the inclusion

$$
\left(x_{p}-\left(\alpha_{p} / \alpha_{r}\right) x_{r}, x_{q}-\left(\alpha_{q} / \alpha_{r}\right) x_{r}\right) \in \mathcal{B}_{\xi / \alpha_{r}}\left(\alpha_{p} / \alpha_{r}, \alpha_{q} / \alpha_{r}\right)
$$

for all cases (i)-(vi). Note that $\mathcal{B}_{\xi / \alpha_{r}}\left(\alpha_{p} / \alpha_{r}, \alpha_{q} / \alpha_{r}\right)$ contains the octagon $\mathcal{O}_{\xi / \alpha_{r}}\left(\alpha_{p} / \alpha_{r}, \alpha_{q} / \alpha_{r}\right)$ with vertices

$$
\begin{array}{ll} 
\pm Q^{1}= \pm\left(\frac{\xi}{\alpha_{r}}, \frac{\xi\left(\alpha_{r}-\alpha_{q}\right)}{\alpha_{r}\left(\alpha_{r}-\alpha_{p}\right)}\right), & \pm Q^{2}= \pm\left(\frac{\xi\left(\alpha_{r}+\alpha_{p}\right)}{\alpha_{r}\left(\alpha_{r}+\alpha_{q}\right)}, \frac{\xi}{\alpha_{r}}\right) \\
\pm Q^{3}= \pm\left(-\frac{\xi\left(\alpha_{r}-\alpha_{p}\right)}{\alpha_{r}\left(\alpha_{r}+\alpha_{q}\right)}, \frac{\xi}{\alpha_{r}}\right), & \pm Q^{4}= \pm\left(-\frac{\xi}{\alpha_{r}}, \frac{\xi\left(\alpha_{r}-\alpha_{q}\right)}{\alpha_{r}\left(\alpha_{r}+\alpha_{p}\right)}\right) .
\end{array}
$$

Let us find the abscissae of points of the intersection of $\mathcal{O}_{\xi / \alpha_{r}}\left(\alpha_{p} / \alpha_{r}, \alpha_{q} / \alpha_{r}\right)$ with the line $y=\xi$. The equation of the line through $Q^{1}, Q^{2}$ is

$$
y+\frac{\alpha_{r}+\alpha_{q}}{\alpha_{r}-\alpha_{p}} x-\frac{2 \xi}{\alpha_{r}-\alpha_{p}}=0 .
$$

By putting $y=\xi$ we obtain

$$
R_{x}=\xi \frac{2-\alpha_{r}+\alpha_{p}}{\alpha_{r}+\alpha_{q}}
$$

The equation of the line through $Q^{3}, Q^{4}$ is

$$
y-\frac{\alpha_{r}+\alpha_{q}}{\alpha_{r}+\alpha_{p}} x-\frac{2 \xi}{\alpha_{r}+\alpha_{p}}=0 .
$$

By putting $y=\xi$ we obtain

$$
L_{x}=-\xi \frac{2-\alpha_{r}-\alpha_{p}}{\alpha_{r}+\alpha_{q}}
$$

(i), (ii). Here $x_{r}=0$ and we have the obvious inclusions

$$
\pm(\xi, 0), \pm\left(\left(\alpha_{p} / 2 \pm 1 / 2\right) \xi, 0\right) \in \mathcal{O}_{\xi / \alpha_{r}}\left(\alpha_{p} / \alpha_{r}, \alpha_{q} / \alpha_{r}\right)
$$

(iii) Here $x_{r}=0$ and thus, by symmetry, it is enough to prove

$$
\left(\left(\alpha_{p} / 2 \pm 1 / 2\right) \xi, \xi\right) \in \mathcal{O}_{\xi / \alpha_{r}}\left(\alpha_{p} / \alpha_{r}, \alpha_{q} / \alpha_{r}\right) .
$$

Let us show that $\left(\left(\alpha_{p} / 2 \pm 1 / 2\right) \xi, \xi\right)$ lies on the segment $\left(L_{x}, \xi\right)\left(R_{x}, \xi\right)$. Indeed,

$$
\left(\alpha_{p} / 2-1 / 2\right) \xi-L_{x}=\frac{\left(3-\alpha_{p}\right)\left(1-\alpha_{r}\right)+\left(1-\alpha_{q}\right)\left(1-\alpha_{p}\right)}{2\left(\alpha_{r}+\alpha_{q}\right)} \xi \geq 0
$$


and

$$
R_{x}-\left(\alpha_{p} / 2+1 / 2\right) \xi=\frac{3\left(1-\alpha_{r}\right)+1-\alpha_{q}+\alpha_{p}\left(1-\alpha_{r}\right)+\alpha_{p}\left(1-\alpha_{q}\right)}{2\left(\alpha_{r}+\alpha_{q}\right)} \xi \geq 0 .
$$

Since $\left(L_{x}, \xi\right)\left(R_{x}, \xi\right) \subset \mathcal{O}_{\xi / \alpha_{r}}\left(\alpha_{p} / \alpha_{r}, \alpha_{q} / \alpha_{r}\right)$, case (iii) is settled.

(iv) Here $x_{r}=0$, and thus, by symmetry, it is enough to prove

$$
\left(\left(\alpha_{p} / 2 \pm 1 / 2\right) \xi,\left(\alpha_{q} / 2 \pm 1 / 2\right) \xi\right) \in \mathcal{O}_{\xi / \alpha_{r}}\left(\alpha_{p} / \alpha_{r}, \alpha_{q} / \alpha_{r}\right) .
$$

Since $\left(\alpha_{q} / 2+1 / 2\right) \xi \leq \xi$, case (iii) implies

$$
\left(\left(\alpha_{p} / 2 \pm 1 / 2\right) \xi,\left(\alpha_{q} / 2+1 / 2\right) \xi\right) \in \mathcal{O}_{\xi / \alpha_{r}}\left(\alpha_{p} / \alpha_{r}, \alpha_{q} / \alpha_{r}\right)
$$

Note that

$$
\left|R_{x}\right|-\left|L_{x}\right|=\frac{2 \alpha_{p}}{\alpha_{r}+\alpha_{q}} \geq 0
$$

and therefore the inequalities $\left(\alpha_{p} / 2-1 / 2\right) \xi \geq L_{x}$ and $\left(\alpha_{q} / 2-1 / 2\right) \xi>-\xi$ imply

$$
\left(\left(\alpha_{p} / 2-1 / 2\right) \xi,\left(\alpha_{q} / 2-1 / 2\right) \xi\right) \in \mathcal{O}_{\xi / \alpha_{r}}\left(\alpha_{p} / \alpha_{r}, \alpha_{q} / \alpha_{r}\right) .
$$

The equation of the line through $\left(\left(\alpha_{p} / 2+1 / 2\right) \xi,\left(\alpha_{q} / 2-1 / 2\right) \xi\right)$ and $(\xi, 0)$ is

$$
y-\frac{1-\alpha_{q}}{1-\alpha_{p}} x+\frac{1-\alpha_{q}}{1-\alpha_{p}} \xi=0 .
$$

It intersects $x=0$ at $\left(0,-\xi\left(1-\alpha_{q}\right) /\left(1-\alpha_{p}\right)\right)$. Since $-\xi\left(1-\alpha_{q}\right) /\left(1-\alpha_{p}\right) \geq-\xi$, we obtain

$$
\left(0,-\xi\left(1-\alpha_{q}\right) /\left(1-\alpha_{p}\right)\right) \in \mathcal{O}_{\xi / \alpha_{r}}\left(\alpha_{p} / \alpha_{r}, \alpha_{q} / \alpha_{r}\right) .
$$

Moreover, $(\xi, 0) \in \mathcal{O}_{\xi / \alpha_{r}}\left(\alpha_{p} / \alpha_{r}, \alpha_{q} / \alpha_{r}\right)$ and by convexity,

$$
\left(\left(\alpha_{p} / 2+1 / 2\right) \xi,\left(\alpha_{q} / 2-1 / 2\right) \xi\right) \in \mathcal{O}_{\xi / \alpha_{r}}\left(\alpha_{p} / \alpha_{r}, \alpha_{q} / \alpha_{r}\right) .
$$

(v) By symmetry it is enough to consider the points

$$
\left(\left(\alpha_{p} / 2 \pm 1 / 2\right) \xi,\left(\alpha_{q} / 2 \pm 1 / 2\right) \xi, \xi\right) .
$$

The square with vertices $\left(\left(\alpha_{p} / 2 \pm 1 / 2\right) \xi,\left(\alpha_{q} / 2 \pm 1 / 2\right) \xi\right)$ lies in the square with vertices $\left(\xi\left(\alpha_{p} \pm 1\right) /\left(1+\alpha_{r}\right), \xi\left(\alpha_{q} \pm 1\right) /\left(1+\alpha_{r}\right)\right)$. Therefore, by the convexity of $\mathcal{G}_{p q r}$, it is enough to consider the points

$$
\left(\xi\left(\alpha_{p} \pm 1\right) /\left(1+\alpha_{r}\right), \xi\left(\alpha_{q} \pm 1\right) /\left(1+\alpha_{r}\right), \xi\right) .
$$

Since

$$
\frac{\alpha_{p} \pm 1}{1+\alpha_{r}} \xi-\frac{\alpha_{p}}{\alpha_{r}} \xi=\frac{ \pm \alpha_{r}-\alpha_{p}}{\alpha_{r}\left(1+\alpha_{r}\right)} \xi, \quad \frac{\alpha_{q} \pm 1}{1+\alpha_{r}} \xi-\frac{\alpha_{q}}{\alpha_{r}} \xi=\frac{ \pm \alpha_{r}-\alpha_{q}}{\alpha_{r}\left(1+\alpha_{r}\right)} \xi,
$$

we have to show that

$$
\left(\frac{ \pm \alpha_{r}-\alpha_{p}}{\alpha_{r}\left(1+\alpha_{r}\right)} \xi, \frac{ \pm \alpha_{r}-\alpha_{q}}{\alpha_{r}\left(1+\alpha_{r}\right)} \xi\right) \in \mathcal{O}_{\xi / \alpha_{r}}\left(\frac{\alpha_{p}}{\alpha_{r}}, \frac{\alpha_{q}}{\alpha_{r}}\right)
$$

This is easily seen from the expressions for the coordinates of the vertices of $\mathcal{O}_{\xi / \alpha_{r}}\left(\alpha_{p} / \alpha_{r}, \alpha_{q} / \alpha_{r}\right)$. 
(vi) By symmetry it is enough to consider the points

$$
\left(\left(\alpha_{p} / 2 \pm 1 / 2\right) \xi,\left(\alpha_{q} / 2 \pm 1 / 2\right) \xi,\left(\alpha_{r} / 2 \pm 1 / 2\right) \xi\right) .
$$

Since $\left(\alpha_{r} / 2+1 / 2\right) \xi \leq \xi$ and case $(\mathrm{v})$ is settled, it is enough to consider the points

$$
\left(\left(\alpha_{p} / 2 \pm 1 / 2\right) \xi,\left(\alpha_{q} / 2 \pm 1 / 2\right) \xi,\left(\alpha_{r} / 2-1 / 2\right) \xi\right)
$$

We have

$$
\begin{aligned}
& \left(\frac{\alpha_{p}}{2} \pm \frac{1}{2}\right) \xi-\frac{\alpha_{p}}{\alpha_{r}}\left(\frac{\alpha_{r}}{2}-\frac{1}{2}\right) \xi=\frac{\alpha_{p} \pm \alpha_{r}}{2 \alpha_{r}} \\
& \left(\frac{\alpha_{q}}{2} \pm \frac{1}{2}\right) \xi-\frac{\alpha_{q}}{\alpha_{r}}\left(\frac{\alpha_{r}}{2}-\frac{1}{2}\right) \xi=\frac{\alpha_{q} \pm \alpha_{r}}{2 \alpha_{r}} .
\end{aligned}
$$

It is easy to verify that

$$
\left(\frac{\alpha_{p} \pm \alpha_{r}}{2 \alpha_{r}}, \frac{\alpha_{q} \pm \alpha_{r}}{2 \alpha_{r}}\right) \in \mathcal{O}_{\xi / \alpha_{r}}\left(\frac{\alpha_{p}}{\alpha_{r}}, \frac{\alpha_{q}}{\alpha_{r}}\right)
$$

\section{Properties of $\mathcal{M}_{\xi}$}

Lemma 11. $\xi F \subset \mathcal{M}_{\xi}$.

Proof. By the definition of $\mathcal{M}_{\xi}$ we have to show that $\xi F \subset \mathcal{G}_{p q r}$ for all $p, q, r \in\{1, \ldots, k+1\}$ such that $p<q<r$ and the set $\mathcal{G}_{p q r}$ is defined. Let $\mathbf{x}=\left(x_{1}, \ldots, x_{k}\right)$ be a vertex of the polytope $\xi F$. By the definition of $F$ either

$$
\mathbf{x}= \pm(\xi, 0, \ldots, 0) \quad \text { or } \quad \mathbf{x}= \pm\left(\xi v_{1}, \ldots, \xi v_{i-1}, \xi, 0, \ldots, 0\right)
$$

where $\left(v_{1}, \ldots, v_{i-1}\right)$ is a vertex of $\mathcal{C}_{1}^{i-1}\left(\alpha_{1} / 2, \ldots, \alpha_{i-1} / 2\right)$. Therefore in the case $p, q, r \in\{1, \ldots, k+1\}, p<q<r$ and $r=k+1$, $\mathbf{x}$ is as in Lemma 9 . Thus $\mathbf{x} \in \mathcal{G}_{p q r}$. For $p, q, r \in\{1, \ldots, k\}$ with $p<q<r, \mathbf{x}$ is as in Lemma 10 . Thus also $\mathbf{x} \in \mathcal{G}_{p q r}$ and by the convexity of $\mathcal{G}_{p q r}$ we have $\xi F \subset \mathcal{G}_{p q r}$.

Lemma 12. $\xi F \neq M_{\xi}$ and therefore $V_{k}\left(\mathcal{M}_{\xi}\right)>(k+1) \xi^{k}$.

Proof. We shall find a plane $P$, such that $\xi F \cap P \neq \mathcal{M}_{\xi} \cap P$. Let

$$
P=\left\{\left(x_{1}, \ldots, x_{k}\right) \in \mathbb{R}^{k}: x_{3}=x_{4}=\ldots=x_{k}=0\right\} \text {. }
$$

Then

$$
\xi F \cap P=\left\{\left(x_{1}, \ldots, x_{k}\right) \in \mathbb{R}^{k}:\left(x_{1}, x_{2}\right) \in \mathcal{X}\right\},
$$

where $\mathcal{X}$ is a polygon. Let us find the set $\mathcal{M}_{\xi} \cap P$. By definition, $\mathcal{M}_{\xi}=$ $\bigcap \mathcal{G}_{p q r}$, thus

$$
\mathcal{M}_{\xi} \cap P=\left(\bigcap \mathcal{G}_{p q r}\right) \cap P=\bigcap\left(\mathcal{G}_{p q r} \cap P\right) .
$$

If $r=k+1$, then 
$\mathcal{G}_{p q r} \cap P= \begin{cases}\left\{\left(x_{1}, \ldots, x_{k}\right) \in \mathbb{R}^{k}:\left(x_{1}, x_{2}\right) \in \mathcal{B}_{\xi}\left(\alpha_{1}, \alpha_{2}\right)\right\} & \text { for } p=1, q=2, \\ \left\{\left(x_{1}, \ldots, x_{k}\right) \in \mathbb{R}^{k}:\left|x_{1}\right| \leq \xi\right\} & \text { for } p=1, q>2, \\ \left\{\left(x_{1}, \ldots, x_{k}\right) \in \mathbb{R}^{k}:\left|x_{2}\right| \leq \xi\right\} & \text { for } p=2, q>2, \\ P & \text { for } p, q>2 .\end{cases}$

while if $r \leq k$ then

$$
\mathcal{G}_{p q r} \cap P= \begin{cases}\left\{\left(x_{1}, \ldots, x_{k}\right) \in \mathbb{R}^{k}:\left(x_{1}, x_{2}\right) \in \mathcal{B}_{\xi / \alpha_{r}}\left(\alpha_{1} / \alpha_{r}, \alpha_{2} / \alpha_{r}\right)\right\} & \text { for } p=1, q=2, r>2, \\ \left\{\left(x_{1}, \ldots, x_{k}\right) \in \mathbb{R}^{k}:\left|x_{1}\right| \leq \xi / \alpha_{r}\right\} & \text { for } p=1, q, r>2, \\ \left\{\left(x_{1}, \ldots, x_{k}\right) \in \mathbb{R}^{k}:\left|x_{2}\right| \leq \xi / \alpha_{r}\right\} & \text { for } p=2, q, r>2, \\ P & \text { for } p, q, r>2 .\end{cases}
$$

Therefore

where

$$
\mathcal{M}_{\xi} \cap P=\left\{\left(x_{1}, \ldots, x_{k}\right) \in \mathbb{R}^{k}:\left(x_{1}, x_{2}\right) \in \mathcal{Y}\right\}
$$

$$
\mathcal{Y}=\mathcal{B}_{\xi}\left(\alpha_{1}, \alpha_{2}\right) \cap\left\{\bigcap_{r \in\{3, \ldots, k\}} \mathcal{B}_{\xi / \alpha_{r}}\left(\alpha_{1} / \alpha_{r}, \alpha_{2} / \alpha_{r}\right)\right\} .
$$

Each of the sets $\mathcal{B}_{\xi / \alpha_{r}}\left(\alpha_{1} / \alpha_{r}, \alpha_{2} / \alpha_{r}\right)$ is centrally symmetric, convex and lies in the square $\mathcal{S}_{\alpha_{r}}=\mathcal{C}_{2 \xi / \alpha_{r}}^{2}(0,0)$. Further, the boundary of each of these sets consists of two horizontal segments on the opposite sides of $\mathcal{S}_{\alpha_{r}}$, two vertical segments on the opposite sides of $\mathcal{S}_{\alpha_{r}}$ and four curvilinear arcs. Analogously, $\mathcal{B}_{\xi}\left(\alpha_{1}, \alpha_{2}\right)$ is centrally symmetric, convex and lies in the square $\mathcal{S}_{1}=\mathcal{C}_{2 \xi}^{2}(0,0)$. Its boundary consists of two horizontal segments on the opposite sides of $\mathcal{S}_{1}$, two vertical segments on the opposite sides of $\mathcal{S}_{1}$ and four curvilinear arcs. Thus, the boundary of any set on the right hand side of (9) consists of segments on the sides of corresponding squares and curvilinear arcs. The number of these sets is finite. Moreover,

$$
\mathcal{S}_{1} \subset \mathcal{S}_{\alpha_{k}} \subset \mathcal{S}_{\alpha_{k-1}} \subset \ldots \subset \mathcal{S}_{\alpha_{3}} .
$$

Thus, if $\mathcal{Y}$ is a polygon then $\mathcal{Y}=\mathcal{S}_{1}$. But by Lemma 2 of [1], $\mathcal{B}_{\xi}\left(\alpha_{1}, \alpha_{2}\right) \neq \mathcal{S}_{1}$, hence $\mathcal{Y} \neq S_{1}$ and thus $\mathcal{X} \neq \mathcal{Y}$. Therefore $\xi F \cap P \neq \mathcal{M}_{\xi} \cap P$ and, finally, $\xi F \neq M_{\xi}$.

11. Proof of the main theorem. Let

$$
\xi=\frac{2}{\left((k+1) n_{k+1}\right)^{1 / k}} .
$$

Then by Lemma 12 there exists $\varepsilon>0$ such that

$$
V_{k}\left(\mathcal{M}_{\xi-\varepsilon}\right)>2^{k} / n_{k+1},
$$

where $\mathcal{M}_{\xi-\varepsilon}$ is defined on replacing $\xi$ by $\xi-\varepsilon$ in the definition of $\mathcal{M}_{\xi}$.

Consider the $(k+1)$-dimensional cylinder $\mathcal{D}$ with base $\mathcal{M}_{\xi-\varepsilon}$ and axis

$$
\left(\tau \alpha_{1}, \ldots, \tau \alpha_{k}, \tau\right), \quad \tau \in\left(-n_{k+1}, n_{k+1}\right) .
$$


Then $\mathcal{D}$ is a centrally symmetric, convex set. By (10),

$$
V_{k+1}(\mathcal{D})=2 n_{k+1} V_{k}\left(\mathcal{M}_{\xi-\varepsilon}\right)>2^{k+1} .
$$

In virtue of Minkowski's first theorem, the set $\mathcal{D}$ contains a non-zero integer vector $\mathbf{m}=\left(m_{1}, \ldots, m_{k+1}\right)$. By (11) we find $\left|m_{k+1}\right|<n_{k+1}$. Therefore in view of condition $\operatorname{gcd}\left(n_{1}, \ldots, n_{k+1}\right)=1$, the vectors $\mathbf{m}$ and $\mathbf{n}$ are linearly independent.

Consider the hyperplane $H\left(x_{k+1}=m_{k+1}\right)$ with induced coordinates $x_{1}, \ldots, x_{k}$. On this hyperplane the point $\left(m_{1}, \ldots, m_{k}\right)$ lies in the translation of $\mathcal{M}_{\xi-\varepsilon}$ with center at $\left(m_{k+1} \alpha_{1}, \ldots, m_{k+1} \alpha_{k}\right)$ and therefore the point $\left(\delta_{1}, \ldots, \delta_{k}\right)$, where $\delta_{1}, \ldots, \delta_{k}$ are defined by (3), lies in $\mathcal{M}_{\xi-\varepsilon}$. Consider the polygon $\mathcal{P}$ defined by (2). By the definition of $\mathcal{M}_{\xi-\varepsilon}$,

$$
\Delta(\mathcal{P}) \geq \frac{1}{n_{k+1}(\xi-\varepsilon)}>\frac{1}{n_{k+1} \xi} .
$$

$\mathcal{P}$ has anomaly 1 (see $[4$, Ch. $3, \S 18$, Theorem 5$]$ ). In virtue of Minkowski's second theorem there exist two linearly independent integer vectors $\left(x_{1}, y_{1}\right)$ and $\left(x_{2}, y_{2}\right)$ such that

$$
\left|m_{i} y_{j}-n_{i} x_{j}\right| \leq \lambda_{j}, \quad i \in\{1, \ldots, k+1\}, j \in\{1,2\}
$$

and

$$
\lambda_{1} \lambda_{2} \Delta(\mathcal{P}) \leq 1
$$

Put

$$
\mathbf{p}=y_{1} \mathbf{m}-x_{1} \mathbf{n}, \quad \mathbf{q}=y_{2} \mathbf{m}-x_{2} \mathbf{n} .
$$

Then $\mathbf{p}, \mathbf{q}$ are linearly independent, and by Theorem 2 of [7] we can assume without loss of generality that

$$
\mathbf{n}=u \mathbf{p}+v \mathbf{q}, \quad u, v \in \mathbb{Z} .
$$

Finally, by (12)-(14) we obtain

$$
h(\mathbf{p}) h(\mathbf{q}) \leq \lambda_{1} \lambda_{2}<n_{k+1} \xi=\frac{2}{(k+1)^{1 / k}} n_{k+1}^{1-1 / k}=\frac{2}{(k+1)^{1 / k}}(h(\mathbf{n}))^{1-1 / k} .
$$

Acknowledgments. The author is deeply indebted to Professor Andrzej Schinzel for helpful discussions, suggestions, advice and guidance.

\section{References}

[1] I. Aliev, On a decomposition of integer vectors, I, Bull. Polish Acad. Sci. Math. 49 (2001), 151-157.

[2] H. G. ApSimon, The critical lattices of the off-centre hypercube, Quart. J. Math. Oxford Ser. (2) 4 (1953), 204-209.

[3] S. Chaładus and A. Schinzel, A decomposition of integer vectors, II, Pliska Stud. Math. Bulgar. 11 (1991), 15-23.

[4] P. M. Gruber and C. G. Lekkerkerker, Geometry of Numbers, North-Holland, 1987. 
[5] K. Mahler, On the minimum determinant and circumscribed hexagons of a convex domain, Proc. Konink. Nederl. Akad. Wetensch. 50 (1947), 692-703.

[6] A. Schinzel, Reducibility of lacunary polynomials, VII, Monatsh. Math. 102 (1986), 309-337.

[7] —, A decomposition of integer vectors, I, Bull. Polish Acad. Sci. Math. 35 (1987), 155-159.

Institute of Mathematics

Polish Academy of Sciences

P.O. Box 137

00-950 Warszawa, Poland

E-mail: iskander@impan.gov.pl

Received on 2.3.2001

and in revised form on 28.8.2001 